of data that have been dismissed by Malawian colleagues due to their unexpectedness. Data, Biruk notes, can do important work. The author's aim is not to expose the uncertainty of numbers (though this is certainly a by-product of the ethnography) but instead to account for what, beyond counting, numbers actually accomplish.

Biruk brings to her account the lens of critical global health studies, which exposes the uneven power relations that many researchers rely upon and (re)produce, and which shapes the expectations, identities and relationships that emerge as a result. Chapter 1 considers how Malawian co-principal investigators are often taken on by foreign researchers in a manner that is "additive, rather than substantive" (50) and that overworks and undervalues Malawian social scientists. Chapter 2 observes that boundaries between Malawian fieldworkers and foreign researchers are constructed through the arena of "local knowledge," through which fieldworkers are attributed expertise only where this status stems from their cultural difference. Biruk argues that Malawian fieldworkers are instead "knowledge workers" (67) whose innovations are essential for survey research to take place. Chapter 3 is also couched within a sharp critique of global inequalities, focused here on the ethical foundations of gift-giving and informed consent processes. These standard practices provide an alibi of clean data and enable researchers to escape unaccountable for the impacts of data collection.

The book also details the ways in which training and expectations for professional comportment and standardised data collection produces social boundaries between Malawian fieldworkers and their research subjects, discussed in Chapters 2 and 4 (for fieldworkers) and Chapter 3 (for research subjects). In this way, just as fieldworkers shape the data, so too does the data shape fieldworkers' identities, imaginations, hopes and anxieties. Participants, who often do not fit the ideal model of altruistic research subjects, also carefully inventory and calculate the benefits of participation. At times, they deliver sharp critiques of vampiristic "bloodsucking," which indexes their dissatisfaction with the extractive logics of research projects.

Cooking Data is a sharply written, meticulously organised and creatively argued book. The volume raises important questions about research ethics and the impacts of enumeration while also adding to the burgeoning study of African intermediaries. A commitment to the lens of critical global health, however, means that the cultural elements of the research context are left unelaborated and, one might argue, undertheorised. Still, Biruk gives enough of a nod to context to demonstrate that her rejection of cultural explanations is a deliberate strategy to assert anthropology's value outside of the "savage slot" of cultural expertise, to demolish rather than reify tropes of global and local, and to tell a different kind of story about the worlds that global health creates. This position is outlined with force in the volume's conclusion, where the tone of the text changes to one that is at once outspoken and inward-facing

Drawing on Black feminist theory, Biruk recognises that the positions she articulates in Cooking Data are held by others whose interpretive labour is less recognised in academic circles. She also calls attention to the fact that public health researchers often fail to bring their results to community stakeholders, thus reproducing the asymmetries global health seeks to redress. In these ways, Cooking Data is more than an ethnography of demographic practice: it is a glimpse into the worlds that enumeration brings into being. It is also a forceful call for all researchers, especially anthropologists, to be more expansive in considering field ethics, more mindful in their participation in asymmetrical relations and more useful in their contributions to global health.

Éric Fassin et Marta Segarra (dir.), Représentation et non-représentation des Roms en Espagne et en France. Paris, Éditions de la Sorbonne, 2018, 311 pages.

\section{Marie Boglari Université Saint-Paul}

Alors que les Roms ${ }^{1}$ sont à la fois objets de politiques étatiques et objets de recherche en sciences sociales, il est nécessaire de réfléchir à la manière dont l'image et la place passive qui leur est accordée entretient l'antitsiganisme européen. Cet ouvrage sur les représentations des Roms en Espagne et en France contient huit articles, écrits par des auteurs Roms et non-Roms, qui sont soit chercheurs, artistes, et membres de la société civile. Chaque auteur discute des défis rencontrés par cette communauté hétérogène quant aux représentations stéréotypiques et aux processus d'objectification et d'essentialisation. Au fil des articles émerge une vue d'ensemble sur la multiplicité des trajectoires de vies et des luttes quotidiennes des Roms.

Dans le chapitre introductif, Éric Fassin souligne l'ambivalence de la représentation, qui a la capacité de présenter tant par une présence qu'une absence. Fassin décrit le portrait raciste des Roms dépeint par les milieux politiques, juridiques, et médiatiques français, lequel occulte la violence symbolique subie par la communauté. La survisibilité répressive des Roms en tant qu'objet de politiques est encouragée par leur invisibilisation juridique et médiatique. Lauteur fait état de la lutte que mènent les minorités en France pour modifier le paradigme de l'invisibilité vers l'audible et briser le "silence complice » (16). L'ouvrage dans son ensemble contribue à ce mouvement et s'articule autour de deux questions : comment représenter sans objectifier, et comme prendre la parole (et la plume) pour sortir de l'invisibilité?

Fassin reproduit ensuite la préface qu'il a rédigée pour l'ouvrage de photographies de Gabriela Lupu, ainsi que certaines de ses œuvres. Fassin révèle d'abord le processus d'abstraction effectué par Lupu (dessins de scènes quotidiennes de personnes Roms mis en scène dans un théâtre de papier, puis photographiés et publiés dans un livre qui n'est plus produit). Fassin réfléchit alors au refus social de voir les Roms, même suite à une telle mise en abîme par Lupu. La distance (ou l'absence directe) des Roms ne suffit pas : même leur «présence spectrale » (30, 40) demeure trop réelle et trop insupportable. Ce constat rejoint l'argumentaire de Marta Segarra concernant la représentation des Roms dans les médias espagnols. En s'appuyant sur une analyse de deux émissions télévisées espagnoles, Segarra affirme que l'invisibilisation des personnes Roms s'opère à travers la construction d'une sur-représentation caricaturale et dévalorisante du groupe. Linteraction entre l'émission et le regard du public permet de cantonner les Roms dans un monde parallèle et de légitimer leur infériorisation. La naturalisation de la distanciation culturelle devient alors le critère d'admission des Roms dans l'imaginaire collectif de la majorité. 
Soulignant la non-représentation des Roms dans le domaine éducatif européen, Rodrigo Andrés offre une réflexion sur les impératifs moraux que devraient respecter les chercheurs lorsqu'ils écrivent, analysent, et enseignent des pièces de littérature non-Roms qui contiennent des représentations des Roms. Andrés relie la 'taxonomie du regard' (déclinée en regards propre, conscient, trouble, et sale) de José Heredia Maya, premier théoricien littéraire Rom en Espagne, aux recherches sur la capacité de la littérature à susciter l'empathie du lecteur. En vue de conscientiser et responsabiliser auteurs et éducateurs quant aux représentations littéraires des gitans, il enrichit la réflexion sur l'éthique du regard (Rollet 2011) et rappelle comment celui-ci influence la réponse affective des autres observateurs.

Les deux articles suivants mettent en pratique la réflexion théorique sur la responsabilité du regard de l'auteur envers les Roms. Mélikah Abdelmoumen relate le choc ressenti lorsqu'elle franchit la frontière de l'imaginaire social à la réalité de Roms sans domicile fixe en France. De ce choc naît le besoin de témoigner des « silences qui hurlent » (74) et de déconstruire les images figées et essentialisées. Lauteure s'interroge sur la manière de « parler de » (77) à travers les formes littéraires du témoignage personnel et de l'essai autobiographique engagé et de trouver un équilibre entre le désir d'agir et la peur de (re)produire les inégalités existantes. Abdelmoumen choisit de raconter " un nous » (79), c'est-à-dire la complexité d'une relation évolutive, et ce en vue d'éviter l'écueil d'une représentation figée d'un 'eux'.

Dans la même veine, Paloma Gay y Blasco et Liria de la Cruz retracent comment leur rencontre et la découverte du monde de l'autre ont orienté leurs trajectoires de vie et leur rapport à l'anthropologie. Gay y Blasco et de la Cruz constatent que l'informatrice et la chercheuse effectuent toutes deux un travail de recherche et produisent des connaissances ethnographiques sur elles-mêmes, sur l'autre, et sur leurs mondes parallèle (94-95). Leur « approche biographique, réflexive et intime » (95) d'écriture ethnographique réciproque et conjointe transgresse les normes de l'anthropologie 'classique' pour permettre à l'objet de la recherche de devenir non seulement sujet, mais acteur de recherche (et de représentation).

Ismael Cortés aborde la question de la représentation des Roms en Espagne sous un double angle historique et politique. L'auteur souligne d'abord les nombreuses mesures répressives envers les Roms allant du $15^{\text {ème }}$ siècle jusqu'à la démocratisation de l'Espagne 1977. Lauteur fait état de l'écart actuel entre la représentation des Roms dans la société civile et l'absence de représentation dans le système politique démocratique. Cortés observe que le mouvement gitan se concentre actuellement sur les défis de l'intégration plutôt que sur l'obtention de droits civils et politique. Il se demande alors comment sortir du cercle vicieux selon lequel l'absence de représentation engendre la non-participation politique et vice-versa.

Dans le dernier article du dossier, Anina Ciuciu retrace les défis et paradoxes de la représentation des Roms dans l'espace culturel, politique et mémoriel français. Ciuciu explore les effets aliénants tant de l'assimilation (toujours impossible) que de la soumission aux stéréotypes des gadjé. L'auteure décrie le statut social auquel la société française limite les Roms en les dépeignant comme "mineurs ", "minorité ", " objets de savoir et de pouvoir ", citoyens sans souveraineté, et «indigènes de l'intérieur» $(112-114,117)$. Ciuciu propose alors que les Roms s'approprient une autoreprésentation de résistants en revisitant leur histoire et leur mémoire, et se dotent d'outils politico-juridiques pour résister aux multiples formes de violence étatique subies au quotidien en France.

Cet ouvrage constitue une contribution importante à l'étude de l'antitsiganisme car il illumine les mécanismes individuels, collectifs, et institutionnels par lesquels la société majoritaire construit une image culturelle figée des Roms, et par la même occasion fixe leur invisibilité et leur impuissance politico-juridique. Chaque auteur soulève une forme différente de " racisme sans race » (Relations 2013) qui cause des dénis de reconnaissance (Renault 2004), que ce soit par des émissions télévisées qui naturalisent un style de vie cliché en une réalité parallèle immuable (45), ou par la bouche des politiciens qui laissent sous-entendre le caractère héréditaire du mode de vie criminel des Roms (14). À la suite de Fanon (1991), les auteurs affirment que la réappropriation du contrôle de l'image - et de l'identité passe par la modification des structures sociales et politicojuridiques. À cet effet, les articles démontrent l'importance de continuer à réfléchir à l'impact de notre regard, de nos mots et de nos pratiques sociales et professionnelles pour développer de nouvelles approches théoriques et pratiques axées sur la participation active des Roms, en tant que communauté et en tant qu'individus, à la construction de leur histoire, de leur image.

La variété des auteurs et des approches ne permet pas de dégager une réflexion approfondie sur le rôle des sciences sociales dans la lutte contre le racisme et les inégalités sociales. Pourtant, cet ouvrage est un pas nécessaire dans cette direction, car il a le mérite de relier une analyse théorique à des exemples d'engagements sociopolitiques et de privilégier le dialogue avec les Roms plutôt que de les figer dans le texte.

\section{Note}

1 Il est à noter que les auteurs utilisent des qualificatifs multiples tels " Gitanxs », " Gitans ", et " Rroms ». Le terme Rom est cependant utilisé ici afin d'alléger le texte.

\section{Références}

Relations. 2013. «Un racisme sans races : Entrevue avec Étienne Balibar ». Relations, 763 : 13-17.

Fanon, Frantz, 1991. Les damnés de la terre. Paris, Gallimard. Renault, Emmanuel, 2004. L'expérience de l'injustice : Reconnaissance et clinique de l'injustice. Paris, La Découverte.

Rollet, Sylvie, 2011. Une éthique du regard: Le cinéma face à la catastrophe, d'Alain Resnais à Rithy Pan. Paris, Hermann.

\section{Roth, Solen. Incorporating Culture: How Indigenous People Are Reshaping the Northwest Coast Art Industry. Vancouver: UBC Press, 2018, 240 pages.}

Thomas McIlwraith University of Guelph

Incorporating Culture describes the business connections and social networks that link northwest coast artists, communities and markets. This is a story of capitalism: a capitalism 